\title{
PSYLLOTYLENCHUS CHABAUDI, N. SP. (Nematodea, Tylenchida : Allantonematidae), parasite de Nosopsyllus fasciatus (Bosc) (Siphonaptera : Ceratophyllidae)
}

\author{
J. DEUNFF* ${ }^{*}$ et H. LAUNAY**
}

RÉSUMÉ. Les auteurs décrivent une nouvelle espèce de Nématode entomogène : Psyllotylenchus chabaudi n. sp. (Tylenchida : Allantonematidae) parasite de Nosopsyllus fasciatus (Bose) (Siphonaptera : Ceratophyllidae), puce du rat, accidentellement présente sur Oryctolagus cuniculus. La nouvelle espèce d'Allantonematidae présente en particulier une volumineuse glande préutérale. Une comparaison des espèces du genre Psyllotylenchus actuellement considérées comme valides est donnée dans la diagnose.

\section{Psyllotylenchus chabaudi, n. sp. (Nematodea, Tylenchida : Allantonema- tidae), parasite of Nosopsyllus fasciatus (Bose) (Siphonaptera : Ceratophyl- lidae)}

SUMMARY. The authors describe a new species of entomogenous Nematoda: Psyllotylenchus chabaudi n. sp. (Tylenchida : Allantonematidae) parasite of Nosopsyllus fasciatus (Bosc) (Siphonaptera : Ceratophyllidae), a rat flea, accidentally present on Oryctolagus cuniculus.

The new species of Allantonematidae presents in particular a very large preuteral gland.

A comparison between the species of the genus Psyllotylenchus which are actually considered as valid is given in the diagnosis.

Dans le cadre d'une action pluridisciplinaire menée en faveur du lapin en France, nous avons entrepris l'étude des vecteurs puces et des mesures prophylactiques ont été envisagées ${ }^{1}$; parmi ces mesures, la lutte biologique contre le siphonaptère du lapin le plus répandu, Spilopsyllus cuniculi, est possible : en effet, il est parasité par trois tylenchides Allantonematidae qui bloquent l'oogénèse chez les puces femelles (Launay et coll., 1983 ; Launay et Deunff, 1983). Au cours de ces enquêtes, l'un de nous (H. L.) a récolté sur Oryctolagus cuniculus des puces appartenant à une espèce normalement inféodée aux muridés : Nosopsyllus fasciatus (Bosc).

* Laboratoire de Parasitologie (Entomologie médicale), Faculté de Médecine, avenue du Professeur-LéonBernard, F 35043 Rennes Cedex.

** Institut de Parasitologie de l'Ouest, même adresse.

I. Travaux effectués grâce à l'aide financière de l'A.N.C.L.A.T.R.A. et à des subventions de l'Office National de la Chasse.

Accepté le 28 juillet 1983 . 
La présence de $N$. fasciatus sur le lapin de garenne est rare et toujours accidentelle et s'explique ici par la cohabitation étroite de lapins et de Rattus sp. dans des garennes installées dans une haie de cannes de Provence, dont la texture du sol était de type sablonneux.

L'intérêt essentiel de cette capture est la découverte d'un nématode nouveau (tylenchide) parasite de $N$. fasciatus, produisant un blocage de l'oogénèse ayant probablement un retentissement sur l'évolution des populations dans cette espèce de puce.

Il s'agit de tylenchides Allantonematidae caractérisées par :

- la présence de générations de femelles gamogénétiques et parthénogénétiques parasites, toutes deux ovovivipares ;

- les deux types de femelles ont une même courbure dorsale, face ventrale vers l'extérieur ;

- la présence d'une glande préutérale volumineuse.

Ces tylenchides appartiennent donc au genre Psyllotylenchus Poinar et Nelson, 1973.

\section{Matériel et Méthodes}

a) Matériel :

- Holotype : femelle parasite gamogénétique

- Allotype : mâle adulte libre

- Paratypes : 50 femelles parasites parthénogénétiques

9 femelles gamogénétiques

2 larves femelles libres de stade IV

34 mâles adultes libres

Tous ces spécimens ont été obtenus directement ou à partir d'élevages de Nosopsyllus fasciatus et sont déposés dans les Collections du Laboratoire de Parasitologie (Entomologie médicale) des U.E.R. médicales et pharmaceutiques de Rennes. Holotype, allotype et une femelle parasite parthénogénétique paratype seront ultérieurement déposés au Laboratoire des vers du Muséum National d’Histoire Naturelle de Paris.

Nous avons l'honneur de dédier ce taxon au Professeur A. Chabaud du Muséum National d'Histoire Naturelle, que nous remercions très vivement de son accueil et de ses précieux conseils.

b) Méthodes :

Les techniques de fixation et de coloration ont été décrites en détails dans un précédent article (Launay, Deunff et Bain, 1983). 


\section{Description}

- femelles parasites gamogénétiques $(n=9)$

Courbées dorsalement, ovovivipares. Longueur : moyenne $516 \mu \mathrm{m}$ (478 à 556), déviation standard 32, Holotype : $507 \mu \mathrm{m}$. Largeur maxima : $43 \mu \mathrm{m}$ (37 à 51), D.S. $=5$, Holotype : $37 \mu \mathrm{m}$. Distance de l'apex au pore excréteur : $14 \mu \mathrm{m}$ (12 à 17), D.S. $=1,6$, Holotype : $16 \mu \mathrm{m}$. Longueur de la queue : $17 \mu \mathrm{m}(13$ à 21$)$, D.S. $=2,8$, Holotype : $16 \mu \mathrm{m}$; queue pointue, régulièrement effilée, pas de mucron visible (fig. 2). Distance de la vulve à l'extrémité postérieure : $34 \mu \mathrm{m}$ (32 à 39$)$, D.S. $=2,9$, Holotype : $35 \mu \mathrm{m}$; vulve sans lèvres visibles. Ovaire antérieur, replié plusieurs fois sur lui-même, spermathèque arrondie, située aux environs du $1 / 3$ antérieur du corps. Les larves peu nombreuses (2 à 4) sont aussi longues que l'utérus, dès ce stade l'ébauche génitale ( 4 cellules vers la moitié du corps) est bien visible. Stylet net, 14 à $15 \mu \mathrm{m}$, en forme de flèche avec un élargissement de la région postérieure ( fig. 2).

- femelles parthénogénétiques $(n=50)$

Courbées dorsalement, ovovivipares. Longueur : $351 \mu \mathrm{m}$ (245 à 436). D.S. $=40$. Largeur maxima : $53 \mu \mathrm{m}$ (31 à 73), D.S. $=9$. Distance de l'apex au pore excréteur : $18 \mu \mathrm{m}$ (14 à 23). Longueur de la queue : $15 \mu \mathrm{m}$ (12 à 17), D.S. $=1,36$; queue conique, large, parfois arrondie, sans mucron visible ( fig. 1). Distance de la vulve à l'extrémité postérieure : $40 \mu \mathrm{m}$ ( 35 à 46$),$ D.S. $=3$; vulve avec des lèvres souvent bien marquées. Ovaire très antérieur, replié plusieurs fois sur lui-même, glande préutérale le plus souvent sphérique, toujours volumineuse : $47 \mu \mathrm{m}$ (30 à 73). Larves plus nombreuses que chez les femelles gamogénétiques (6 à 12), leur ébauche génitale n'est pas visible (fig. 1). Stylet de très petite taille, filiforme, parfois peu visible : 6 à $8 \mu \mathrm{m}$.

- larves femelles libres $L 4(n=2)$

Habitus rectiligne, longueur $340 \mu \mathrm{m}$, largeur 18-20 $\mu \mathrm{m}$, longueur de la queue 22-26 $\mu \mathrm{m}$, distance ébauche du vagin à l'extrémité postérieure : $48 \mu \mathrm{m}$, ovaire composé de 4 à 5 cellules, stylet présent mais très réduit ( fig. 3,4 et 5).

- mâles adultes libres ${ }^{2}(n=34)$

Courbés ventralement, rarement rectilignes avec toujours l'extrémité caudale courbée en crosse ventralement. Longueur : $321 \mu \mathrm{m}$ (269 à 389), D.S. $=29$, allotype : $389 \mu \mathrm{m}$. Largeur maxima : $16 \mu \mathrm{m}$ (12 à 20$),$ D.S. $=1,8$, allotype : $16 \mu \mathrm{m}$. Distance de l'apex au pore excréteur : $21 \mu \mathrm{m}$ (16 à 25$),$ D.S. $=2,3$, allotype : $24 \mu \mathrm{m}$. Distance de l'apex à l'anneau nerveux : $39 \mu \mathrm{m}$ (33 à 46$),$ D.S. $=4$. Queue trappue, extrémité arrondie : $21 \mu \mathrm{m}$ (19 à 25), D.S. $=1,2$, allotype : $23 \mu \mathrm{m}$. Spicules arqués, longueur

2. Obtenus en élevage. 


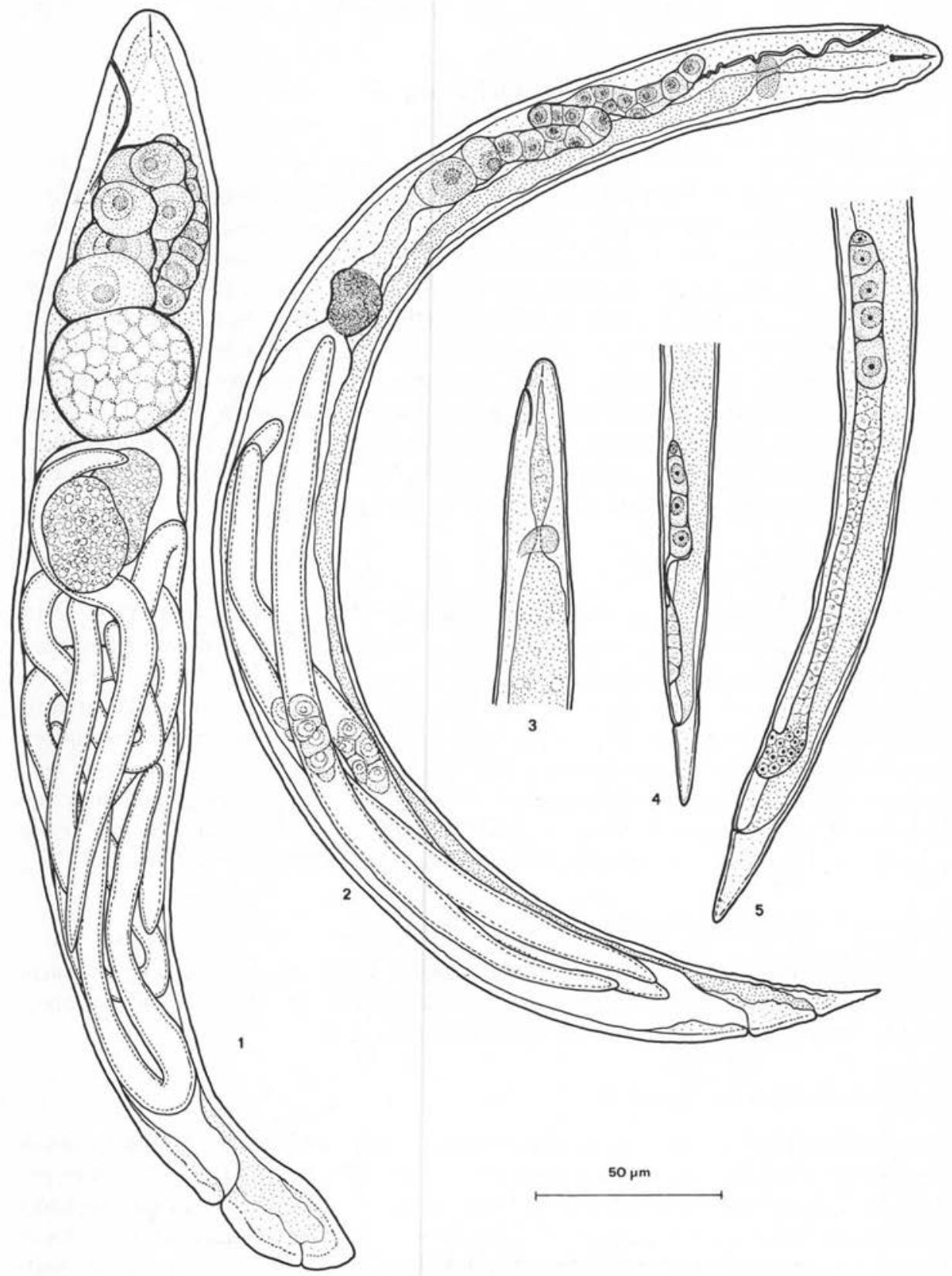

FIG. I à 5. - Psyllotylenchus chabaudi sp. n.

$I$ - femelle parthénogénétique, paratype, vue latérale gauche ;

2 - femelle gamogénétique, holotype, vue latérale gauche ;

3, 4, 5-larves femelles libres de stade IV ;

3 - partie antérieure ;

4 - stade IV précoce, partie postérieure ;

$s$ - stade IV âgé, partie postérieure. 
12 à $16 \mu \mathrm{m}$, allotype : $15 \mu \mathrm{m}$. Gubernaculum peu développé, bursa pélodère, ailes peu marquées. Stylet réduit, grêle, longueur 4 à $6 \mu \mathrm{m}$ (fig. 6 à 9).

- Hôte type: Nosopsyllus fasciatus Bosc 1802

- Localité type : Pernes-les-Fontaines (Vaucluse, France), octobre 1979.

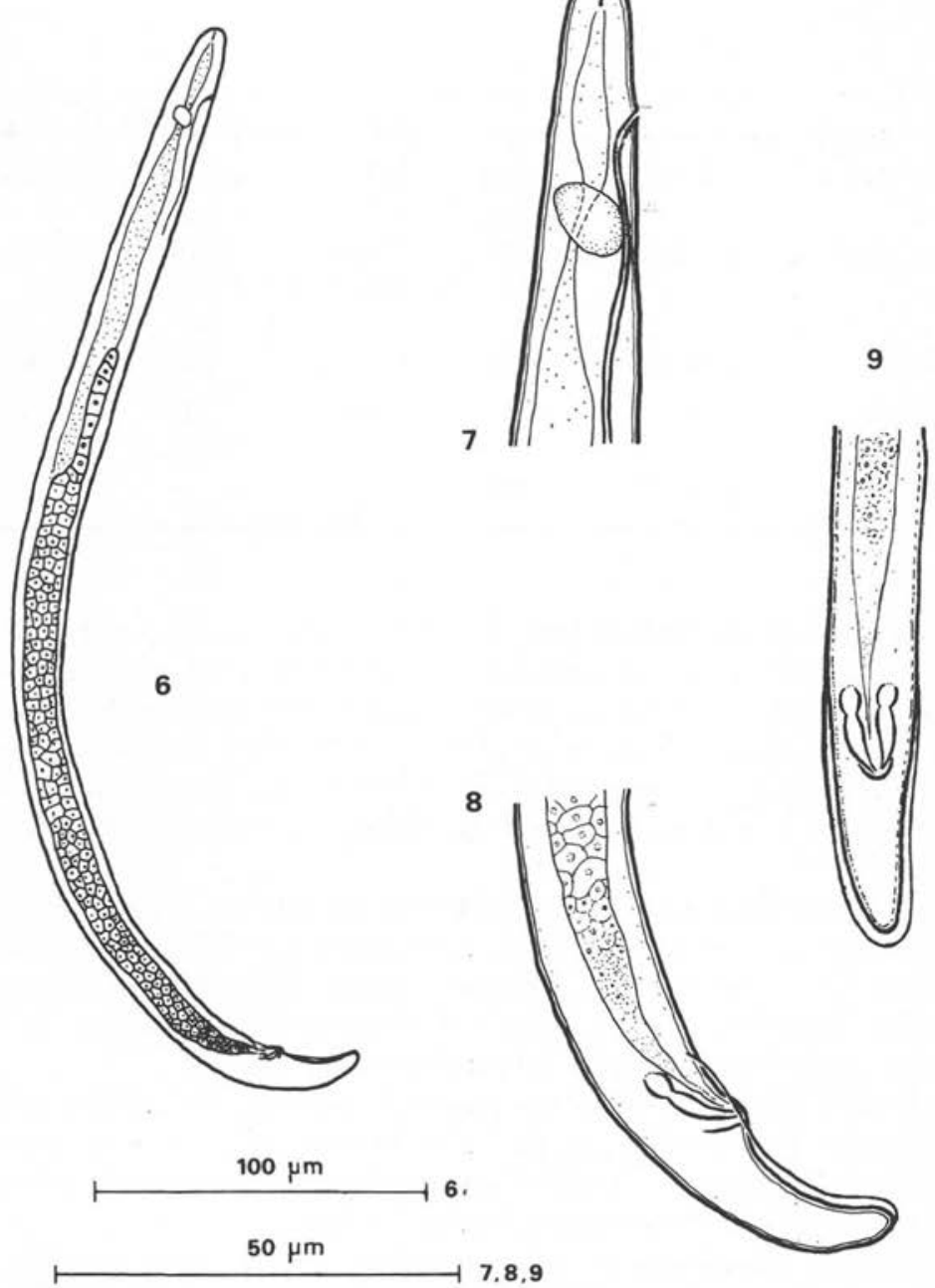

FIG. 6 à $9-$ Psyllotylenchus chabaudi sp. n., mâle adulte libre allotype 6 - habitus ; 7 - partie antérieure ;

8 - partie postérieure, vue latérale droite; 9 - partie postérieure, vue ventrale. 


\section{Diagnose}

Actuellement $P$. chabaudi n. sp. doit être séparé des seules espèces suivantes : P. viviparus Poinat et Nelson, 1973, P. pawlowskyi (Kurochkin, 1960), P. koslovi Rubtzov et Tshumatova, 1981*, P. caspius Rubtzov et Samurov, 1981*, P. latifrons Rubtzov et Tshumatova, 1981*, P. neopsyllus Rubtzov et Tshumatova, 1981* (tableau I).

TABleaU I. - Comparaison des mensurations des espèces du genre Psyllotylenchus.

\begin{tabular}{|c|c|c|c|c|c|c|c|c|}
\hline & & viviparus & pawlowski & koslovi & caspius & latifrons & neopsyllus & chabaudi \\
\hline \multirow{3}{*}{ 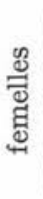 } & gamo. & $840-1480 \mu \mathrm{m}$ & $2400-3100$ & 1460 & 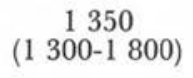 & 840 & 500 & 478 à 556 \\
\hline & $\begin{array}{l}\text { par- } \\
\text { théno. }\end{array}$ & $360-840$ & $600-820$ & 530 & $450-500$ & 370 & 400 & 245 à 436 \\
\hline & stylet & $15-20$ & 22 & $16-17$ & 15 & 10 & 10 & $14-15$ \\
\hline & mâles & $620-740$ & $300-550$ & 156 & & & & 269 à 389 \\
\hline
\end{tabular}

Nous ne pouvons pas tenir compte des autres espèces décrites par Rubtzov dans ce genre car elles sont, non seulement insuffisamment décrites, mais de plus l'auteur ne donne aucun argument en faveur de leur appartenance au genre Psyllotylenchus.

Les femelles gamogénétiques de $P$. chabaudi se séparent facilement, par la taille, de celles de $P$. viviparus, $P$. pawlowskyi, $P$. koslovi, $P$. caspius et $P$. latifrons. De plus chez $P$. neopsyllus, $P$. koslovi, $P$. latifrons la vulve est subterminale et donc différente de P. chabaudi.

Les femelles parthénogénétiques se séparent non seulement par la taille de celles de $P$. pawlowskyi, $P$. koslovi, P. caspius mais encore par la forme (nettement piriforme) de celles de $P$. viviparus, $P$. koslovi, $P$. caspius, $P$. latifrons, $P$. neopsyllus.

La taille des mâles permet de séparer $P$. chabaudi de $P$. viviparus et $P$. koslovi, en outre chez viviparus la queue est très effilée.

Enfin la taille du stylet permet de séparer $P$. chabaudi de $P$. pawlowskyi, $P$. koslovi, $P$. latifrons et $P$. neopsyllus. De plus chez $P$. neopsyllus, $P$. caspius, $P$. latifrons, la glande préutérale est petite, d'après Rubtzov ( $o p$. cit.), ce qui n'est pas en accord, d'ailleurs, avec les caractères du genre Psyllotylenchus...

En conclusion nous estimons qu'actuellement dans le genre Psyllotylenchus, il y a 3 espèces présentant les caractères du genre sensu stricto $: P$. viviparus, $P$. parvlowskyi et $P$. chabaudi. Ces 3 espèces sont nettement séparées par leurs dimensions

* In Rubtzov, 198r. 
mais très homogènes dans leurs structures. En particulier, elles possèdent toutes les trois une glande préutérale sphéroïde volumineuse. La diagnose des espèces décrites par Rubtzov sera probablement à revoir.

\section{Biologie - Phénologie}

Le cycle de $P$. chabaudi est identique à celui des autres espèces du même genre. Les mâles et femelles libres s'accouplent dans le milieu extérieur et seules les femelles fécondées vont pénétrer chez l'hôte. Ces femelles gamogénétiques augmentent alors de taille, elles sont ovovivipares et libèrent des larves peu nombreuses mais de grande taille. Tout ou partie de ces larves donnent des femelles parthénogénétiques $\left(2^{\mathrm{e}}\right.$ génération) également ovovivipares qui vont libérer de nombreuses larves. Ces larves vont sortir de la puce et donner dans le milieu extérieur les mâles et femelles libres.

\section{Spécificité}

Nous considérons $P$. chabaudi comme une espèce très spécifique de Nosopsyllus fasciatus. En effet, dans cette zone où cohabitent rats et lapins, seules les puces de rats $N$. fasciatus ont été trouvées porteuses de $P$. chabaudi, l'examen de 528 Spilopsyllus cuniculi trouvés sur les mêmes lapins s'est révélé négatif. En revanche $S$. cuniculi est connue comme hôte d'autres tylenchides.

\section{Remarque}

Nous tenons à souligner le fait que la zone de capture présente un sol de texture sablonneuse, en effet nous portons actuellement une attention toute particulière à la texture du substrat dans lequel vivent les stades libres de ces nématodes entomogènes. Une étude approfondie de la granulométrie et des caractéristiques édaphiques fera l'objet d'une prochaine publication, mais dès à présent nous constatons que les régions sablonneuses livrent plus fréquemment des puces parasitées que les régions à sol argileux. En fait ce phénomène est bien connu à propos de certains nématodes libres et s'applique donc probablement aux stades libres des nématodes parasites d'insectes qui sont en fait beaucoup plus étroitement liés au substrat qu'à l'hôte (parasité uniquement par les femelles et les larves dans la plupart des cas).

Remerciements. Nous remercions le Docteur Odile Bain de ses encouragements et de son aide dans nos études sur les nématodes entomogènes.

Nous adressons enfin nos vifs remerciements au Professeur Beaucournu qui est à l'origine de nos études sur ces hyperparasites et continue de nous faire profiter de son expérience concernant la systématique et la pathologie des Siphonaptères. 


\section{BIBLIOGRAPHIE}

KUROCHKIN Yj. V. : (The nematode Heterotylenchus pawlowskyi sp. n., castrating plague-carrying fleas). Doklad. Acad. Sci. USSR, 1960, 135, 1281-1284 (en russe) (résumé anglais).

Launay H., Deunff J., BaIN O. : Spilotylenchus arthuri, gen. n., sp. n. (Nematodea, Tylenchida : Allantonematidae), parasite de Spilopsyllus cuniculi (Dale, 1878) (Siphonaptera: Pulicidae). Ann. Parasitol. Hum. Comp., 1983, 58, 14I-150.

LAUNAY H., DeUNFF J. : Un Spilotylenchus nouveau (Nematodea, Tylenchida: Allantonematidae), parasites de Spilopsyllus cuniculi (Dale) (Siphonaptera: Pulicidae), Siphonaptère : inféodé au lapin de garenne. Ann. Parasitol. Hum. Comp. (sous presse).

Poinar G. O., Nelson B. C. : Psyllotylenchus viviparus, n. gen., n. sp. (Nematodea : Tylenchida, Allantonematidae) parasitizing fleas (Siphonaptera) in California. J. Med. Ent., 1973, 10, 349-354.

RubTSOy I. A. : (Parasites and enemies of fleas). Parazity i vragi blokh. Nauka, Leningrad, USSR, I981, I04 p. (en russe) (résumé anglais). 